\title{
Cenplesto
}

\section{Estudo bibliométrico sobre moda em teses e dissertações na área das Ciências da Comunicação}

\author{
Eneus Trindade \\ Doutor; Universidade de São Paulo, São Paulo, SP, Brasil; \\ eneustrindade@usp.br \\ Priscila Rezende Carvalho \\ Doutoranda; Universidade de São Paulo, São Paulo, SP, Brasil; \\ preerezende@gmail.com \\ Francisco Carlos Paletta \\ Doutor; Universidade de São Paulo, São Paulo, SP, Brasil; \\ fcpaletta@usp.br
}

\begin{abstract}
Resumo: A recente institucionalização da moda como objeto de estudo no campo científico e acadêmico e o crescente interesse no assunto observado em diversas áreas são as premissas desta bibliometria de teses e dissertações. $\mathrm{O}$ objetivo é dimensionar e caracterizar a produção tendo como escopo os programas de Pós-Graduação em Comunicação no Brasil, explorando quantitativamente como essa área, em particular, fomentou o debate sobre o assunto na academia. Para tanto, o trabalho passa por duas discussões principais: pela relação entre a moda e os estudos dos consumos na área da comunicação, e pela bibliometria e o uso de ferramentas computacionais para a visualização e comunicação de resultados. O período delimitado para o corpus de análise é entre os anos de 2006 e 2018, em que foi observado crescimento da produção sobre o tema. Além de demonstrar a evolução da produção neste período, os resultados apresentam as instituições e os pesquisadores de destaque e relacionam as palavras-chave mais utilizadas, vislumbrando tendências de pesquisa.
\end{abstract}

Palavras-chave: Organização da Informação. Bibliometria. Teses e Dissertações. Comunicação. Moda.

\section{Introdução}

No Brasil, o interesse de pesquisadores de áreas distintas por investigar assuntos relacionados à moda cresceu nas últimas três décadas, o que resulta em uma produção de conhecimento científico incipiente e dispersa entre as áreas do saber. Isso é demonstrado na análise bibliométrica de Orestes Trevisol Neto (2015), que reuniu trabalhos sobre moda oriundos de programas de diversas 
áreas do conhecimento, entre 1988 e 2013, e incluiu documentos complementares (currículos de professores e materiais dos cursos em que trabalham, apresentações em eventos científicos e publicações em periódicos da área), relacionando a dimensão social (a das instituições) e a dimensão cognitiva (a das produções intelectuais) do desenvolvimento científico, como proposto por Richard Whitley (1974).

O desenvolvimento e a decorrente institucionalização do campo do conhecimento sobre moda foram impulsionados, em grande parte, por dois eventos: o surgimento e a popularização de cursos superiores que visavam atender à demanda por profissionais da cadeia de têxteis e de confecções brasileira nos anos 1980 e a configuração mercadológica do setor, incluindo os segmentos midiáticos, a partir dos anos 1990 (BONADIO, 2010). Portanto, trata-se de um fenômeno relativamente recente.

Apenas três programas de pós-graduação no Brasil têm a moda entre suas linhas de pesquisa principais: o programa de Mestrado Acadêmico em Têxtil e Moda da Escola de Artes, Ciências e Humanidades da Universidade de São Paulo, criado em 2011 ${ }^{1}$, o Mestrado Profissional em Design de Vestuário e Moda, da Universidade do Estado de Santa Catarina, de 2017, e Arte, Moda: História e Cultura, do Programa de Pós-Graduação em Artes, Cultura e Linguagens da Universidade Federal de Juiz de Fora, que desde 2019 oferece também o curso de doutorado. Ou seja, o conhecimento científico necessário para sua legitimação nas instituições de ensino superior é oriundo de outras especialidades e de suas visadas sobre a moda como objeto, a exemplo da interface Comunicação, Moda e Consumo, aqui estudada.

Existe uma produção relevante sobre moda na Comunicação como área de concentração, mas, não há o mapeamento e a caracterização desta produção. É esse o objetivo principal do presente artigo, que recorre às orientações do método bibliométrico para atingir tal intento.

A hipótese trabalhada é a de que a moda no campo acadêmico da comunicação apresenta dispersões tanto na sua institucionalização social quanto cognitiva, não apresentando uma comunidade formada ou paradigmas tradicionais, apesar da trajetória ascendente. 
O interesse em discutir esses resultados é justificado pela necessidade de avaliar estrategicamente os caminhos e as tomadas de decisão no fazer acadêmico para prospectar temas de pesquisa e para analisar colaborações e contribuições, visando ao progresso desta interface em um cenário de conflitos entre temas prioritários, discriminações por áreas e de concorrências por reconhecimento e por legitimidade dos objetos científicos (BOURDIEU, 1983).

Ao passo que o tema se consolida, as iniciativas para documentar e para entender sua trajetória e sua historicidade surgem na intenção de eleger autores e obras que são referências para novos pesquisadores e de indicar as temáticas e as metodologias mais ou menos amadurecidas, sugerindo oportunidades para novos projetos em vertentes pouco exploradas. Outros levantamentos correlatos demonstram que esse tipo de pesquisa pautada pela bibliometria traz contribuições valiosas para aqueles envolvidos com temas emergentes e evidenciam o empenho pelo fortalecimento da pesquisa em moda também em outros países.

$\mathrm{Na}$ Espanha, apesar de “[...] ocupar espaço predominante em âmbito empresarial" (ALARCÓN, 2019, p. 217), uma análise das teses de doutorado publicadas em vinte e quatro anos mostrou um cenário semelhantemente incipiente, com trabalhos exploratórios ou descritivos em distintas linhas de investigação, encontrando-se em uma primeira etapa do desenvolvimento científico, mas, com a diferença de que esses estudos foram realizados em programas próprios da área da Moda e da produção têxtil, indicando menor dispersão.

Tendo este cenário em consideração, realizamos um levantamento de teses e dissertações na base de dados da Coordenação de Aperfeiçoamento de Pessoal de Nível Superior (CAPES), com busca pelo termo moda tendo a Comunicação como área de concentração. Pela bibliometria, a produção do conhecimento nesta interface foi organizada procurando apontar possíveis tendências, sobretudo, no que diz respeito à interface comunicação, moda e consumo.

Neste estudo exploratório e quantitativo, são definidas a dimensão e as características do corpus de análise de uma pesquisa em andamento, 
esclarecendo as decisões tomadas para as próximas etapas. Ademais, os resultados aqui apresentados entregam um mapeamento inédito, baseado em dados, da presença da moda na área da Comunicação, com o potencial de promover novas pesquisas nessa arena interdisciplinar.

\section{A moda e os estudos do consumo nas Ciências da Comunicação}

Para José Luiz Braga (2004), a Comunicação, assim como todas as ciências humanas e sociais, beneficia-se de articulações entre disciplinas em suas elaborações conceituais e metodológicas, articulações essas abordadas pela noção de interface. Nos estudos de interface, as fronteiras não se desfazem a ponto de uma disciplina ser definida pela outra - como na afirmação de que "tudo é comunicação". Pelo contrário, há de se explicitar exatamente o questionamento sobre o objeto que cabe ao campo da Comunicação investigar e que não caberia, portanto, às outras especialidades das ciências humanas e sociais.

$\mathrm{Na}$ intersecção entre as partes, dá-se a construção de problemas de interação que se resolvem, privilegiadamente, pela perspectiva da área comunicacional, mas que "[...] cada componente comparece com seu acervo (historicamente constituído) de práticas e de conhecimentos.” (BRAGA, 2004, p.226). Em outras palavras, são problemas advindos de áreas extra comunicacionais, mas que têm a comunicação como questão.

A moda, no campo científico e acadêmico, apresenta dispersões tanto na dimensão social quanto na dimensão cognitiva, já que é estudada por abordagens “[...] filosóficas, históricas, sociais, comportamentais, econômicas, estéticas e tecnológicas":

A institucionalização cognitiva contempla os objetos de pesquisa, conceitos, teorias, técnicas, métodos, leis e problemas próprios de uma disciplina, materializados na produção bibliográfica de seus pesquisadores. Por sua vez, a institucionalização social refere-se às instituições formais que constituem o próprio campo, abrangendo a comunidade científica, por meio dos grupos de pesquisa, cursos de graduação e pós-graduação, periódicos, eventos/reuniões acadêmicos e associações/sociedades científicas (SILVA; CAFÉ; TREVISOL NETO, 2017, p.136). 
Mesmo se enraizando neste conjunto aparentemente desordenado de objetos, métodos e problemáticas, atualmente, a moda é prestigiada em análises das "[...] dinâmicas sociais, hierarquias, distinção de classe, consumo e questões de gênero, entre outras." (RAINHO; VOLPI, 2018, p.211). Para que atingisse tal estágio de legitimação, contou com o pioneirismo de Gilda de Mello e Souza que, nos anos 1950, apresentou tese de doutoramento sobre o as roupas e a moda no Brasil do século XIX, trabalho publicado em livro apenas 37 anos depois, reconhecimento tardio após ter sido minimizado por seus pares da sociologia, que demonstraram pouco saber sobre a história da moda ao tecerem suas considerações (PRADO, 2019, p.16).

Ao eleger um objeto pouco estabelecido pela produção científica e acadêmica, embora tenha havido avanço, nos expomos a avaliações orientadas pelo senso comum. Por isso, enfatizamos a preocupação em não reduzir a junção dos dois universos a simples troca de dados, em que dois grupos fornecem produtos um ao outro sem serem obrigados a se encontrarem (BOURDIEU; CHAMBOREDON; PASSERON, 1999). Ou seja, sem buscar no objeto adotado o conteúdo teórico e contextual específico.

Comunicação é uma das áreas que se destacam na produção de saberes sobre a moda, como mostrou o mapeamento de trabalhos realizados na pósgraduação stricto sensu feito pela historiadora Maria Claudia Bonadio (2010). Um dos resultados apresentados afirma que a maior parte de teses de doutoramento, na época do estudo, era proveniente de programas de pósgraduação em Comunicação.

Conforme as teorias da comunicação desenvolveram-se, fenômenos do consumo ganharam visadas contrastantes, a depender do paradigma sob o qual se construíam as inter-relações teóricas e empíricas (PERES-NETO, 2016). Nas últimas décadas, pesquisadores da comunicação engajaram-se em tratar o consumo como tema complexo que promove uma reflexão privilegiada sobre as contradições do mundo contemporâneo e da vida cotidiana, com significados plurais que superam o valor pejorativo do consumismo, superam a centralidade em eventos comerciais de compra e venda e o entendimento de audiências como 
passivas aos produtos midiáticos.

Segundo Trindade (2019, p. 115), passam a ser abarcadas “[...] questões de circulação, usos e consumos midiáticos de mídias, marcas, produtos culturais e serviços digitais." Assim, eleva-se o consumo como um subcampo da comunicação, considerando as apropriações individuais dos bens e das mensagens em circulação pelas interações, sejam interações midiáticas ou presenciais.

É nesta esteira de reflexões que o termo consumo vem aplicado na função plural, pois o consumo também tem amplas acepções e tem sido utilizado para ampliar o escopo dos objetos da mediação cultural dos mercados na vida cotidiana [...] (TRINDADE, 2019, p. 118).

Os fenômenos do consumo são "elusivos e ambíguos", de acordo com Lívia Barbosa e Colin Campbell (2006, p. 21). Os autores expõem como essa ambiguidade requer "[...] ênfase simultânea nos sentidos negativo e positivo do consumo", tanto na academia como também nas nossas experiências individuais como consumidores (BARBOSA; CAMPBELL, 2006, p.22). Ao tratar do consumo, lidamos com aspectos não só relacionados ao seu sentido de esgotamento material da sociedade e do meio ambiente, mas, na mesma medida, com aspectos produtivos, criativos e de grande significado cultural.

Nessa direção, uma das proposições mais influentes é o modelo de transmissão de significados culturais pelo consumo sugerido pelo antropólogo Grant McCracken (2003), que destaca tanto a publicidade quanto a moda como sistemas responsáveis pela movimentação de significados entre os bens de consumo e os indivíduos. A movimentação se dá, de acordo com o autor, de forma estruturada por categorias e por princípios culturais transubstanciados em objetos materiais pelos instrumentos da moda e da publicidade, com os quais os indivíduos consumidores interagem por rituais de posse, de troca, de arrumação e de despojamento, defendendo as contribuições do consumo no entendimento da cultura.

Por essas interações se dão os processos comunicacionais da moda, aqueles que organizam o comum, como disposto por Muniz Sodré, desde os vínculos que dão coesão às relações humanas, até àqueles organizados pelas 
tecnologias atuais, pelos quais nos sociabilizamos uns com os outros e com o mundo material do qual participamos (SODRÉ, 2014).

Ainda há a necessidade de construir a historicidade da moda como objeto de tamanha complexidade para a comunicação, explicitando quais problemáticas tem sido investigadas e qual o estado de maturidade para, de forma prospectiva, "[...] abri-lo para dar continuidade à sua construção, pois um saber não é, em essência, nem estático nem definitivo.” (LOPES, 2003, p. 282). Este trabalho contribui com essas lacunas por meio da análise bibliométrica.

\section{Bibliometria, organização e visualização da informação}

Por definição, a bibliometria trata de um conjunto de leis e princípios aplicados a métodos estatísticos e matemáticos que visam ao mapeamento da produtividade científica a partir de descrições de informação e de documentos (CAFÉ; BRÄSCHER, 2008).

O objeto de estudo são documentos que devem estar acessíveis em meios de publicação duráveis e seguros, que sejam recuperáveis e que não exista a previsão de retirada das publicações, gratuitas ou não (BORGMAN; FURNER, 2002). Entre os tipos de produção mais relevantes estão os livros, as teses, os capítulos de livros, os artigos publicados em revistas científicas, as comunicações em altas conferências, os relatórios técnicos, os materiais pedagógicos, os whitepapers e as páginas da web (LOPES et al., 2012).

A partir da análise da comunicação acadêmica é possível vislumbrar tendências de estudos ou o estado da arte de campos específicos e, também, a popularidade de determinados temas (YALE;GILLY, 1988).A disponibilização de sistemas de catalogação e de indexação online facilitou a recuperação de informações científicas e sua aplicação em outras áreas além da biblioteconomia e das ciências da informação, fornecendo conceitos científicos e elementos técnicos para medir a produção, avaliar e tomar decisões em seus respectivos campos (CÔRTES;RODRIGUES, 2016, p.2).

As teses e dissertações são documentos classificados como literatura cinzenta pela "[...] pouca probabilidade de serem adquiridos através de canais usuais de vendas de publicações [...]", por serem a comunicação do resultado do 
processo pelo qual passa um pesquisador que, mesmo sendo avaliado por pares, não tinha, em sua origem, a visibilidade e acessibilidade da literatura comercial (LARA, 2006, p.401). De grande importância para os estudos de objetos emergentes, este tipo de literatura está em maior evidência desde que as tecnologias da informação aprimoraram bibliotecas digitais e bases de dados específicas (FUNARO; NORONHA, 2006).

Conforme Carlos Alberto Araújo (2006), a bibliometria se desenvolve no início do século XX como estudo da mediação e da disseminação do conhecimento, baseada em três leis empíricas do "comportamento da literatura": “[...] o método de medição da produtividade de cientistas de Lotka - 1926, a lei de dispersão do conhecimento científico de Bradford - 1934 e o modelo de distribuição e freqüência de palavras num texto de Zipf - 1949." (ARAÚJO, 2006, p. 12). Mas, desde a década de 1990, são desenvolvidos estudos em que os métodos e as técnicas bibliométricas são aplicadas como suporte instrumental de metodologias qualitativas nas ciências sociais (ARAÚJO, 2006).

A opção por adotar instrumentos oriundos dessa área justifica-se pela sua qualidade de englobar vários indicadores empíricos, entendidos como critérios mensuráveis de observação e análise, tais como "autores, citações e conteúdo textual", encontrados nos registros de teses e dissertações, entre outras formas de comunicação acadêmica, na própria área e entre áreas (BORGMAN, 1989, p.584).

Os indicadores são elementos complexos, unidades de análise com funções particulares e relacionáveis entre si. Indicadores bibliométricos, especificamente, são construídos de acordo com "objetivos, enfoques e aplicações" da bibliometria em cada caso, podendo ser desde o número de publicações - ao medir a produtividade científica - até as coocorrências de palavras no texto, o que demonstra relações entre temas pesquisados neste universo, como explicado por Maricato e Noronha (2013).

Os autores mostram que os exemplos de indicadores formam um imenso conjunto de expressões, quase sempre quantitativas, concebidas para empreender monitoramentos da dinâmica da pesquisa, observações de tendências, mensuração de impacto de artigos, mapeamentos, e tantas outras 
atividades possíveis a partir de documentos acadêmicos. O que faz da bibliometria um conhecimento apropriado para a avaliação do crescimento e do amadurecimento de campos recentes, tal qual é o objetivo do presente estudo.

A junção entre métodos bibliométricos e métodos de visualização da informação oferece soluções para ordenar tal complexidade, permitindo representações gráficas da situação do conhecimento em um dado campo científico, conduzindo novos pesquisadores a temáticas e campos ainda a se desenvolverem, ao exibir a produção e a disseminação do conhecimento (QUEVEDO-SILVA, et al. 2016).

Com o objetivo de ajudar pesquisadores a saberem quais perguntas fazer, ferramentas de visualização da informação oferecem panoramas funcionais para testar hipóteses e compartilhar resultados (BEDERSON; SHNEIDERMAN, 2003). Suas formas de representação comunicam as atividades executadas, detectando paradigmas e fronteiras a serem transpassadas para levar a ciência adiante, demonstrando e ampliando rastros de controvérsias, de crises e de debates a partir da organização e análise de documentos e informações, aplicando a "ciência na própria ciência" (CHEN, 2006).

Neste trabalho, foram utilizadas as ferramentas Iramuteq $^{2}$ e RStudio ${ }^{3}$, ambas interfaces para análises na linguagem R. Apesar disso, os dados foram estruturados e normalizados manualmente pelos autores, que organizaram a informação de acordo com as instruções adequadas para a análise pela ferramenta, sem perder a reflexividade epistêmica, "a vigilância, a crítica e a autorreflexão" também nesta etapa da pesquisa, tomando a informação dada pelas ferramentas como parte da construção do objeto, e não como o objeto em si (LOPES, 2010, p.34-35).

A realidade é que, na prática, os dados nunca estarão do jeito que você de fato precisa. Portanto, é fundamental dominar técnicas de manipulação de dados. Entendamos a manipulação de dados como o ato de transformar, reestruturar, limpar, agregar e juntar os dados. Para se ter uma noção da importância dessa fase, alguns estudiosos da área de Ciência de Dados costumam afirmar que $80 \%$ do trabalho é encontrar uma boa fonte de dados, limpar e preparar os dados, sendo que os $20 \%$ restantes seriam o trabalho de aplicar modelos e realizar alguma análise propriamente dita (OLIVEIRA; GUERRA, McDONNELL, 2018, p.25). 


\section{Etapas e procedimentos metodológicos}

A primeira etapa da pesquisa se dá na revisão teórica acerca da interface investigada (que intersecciona comunicação, moda e consumo) e da reflexão sobre bibliometria e Visualização da Informação enquanto fontes de métodos e técnicas de investigação, como foi exposto nos itens anteriores.

Em seguida, foi feita a formação do corpus e o levantamento de teses e dissertações sobre moda na área das Ciências da Comunicação no Brasil na base de dados da Coordenação de Aperfeiçoamento de Pessoal de Nível Superior (CAPES), cujos dados são abertos e o acesso é on-line. A escolha por teses e dissertações tem o intuito de "[...] rememorar e reavaliar a atividade científica desenvolvida na universidade.” (KOBASHI; DOS SANTOS, 2008, p.108).

A estratégia de busca utilizou apenas o descritor "moda". Os 2.690 resultados apresentados foram refinados pelo critério "Área do Conhecimento", filtro permitido pela plataforma, selecionando a opção "Comunicação" e “Comunicação Visual”, o que retornou 320 títulos. Os demais filtros não foram utilizados.

Após o levantamento, foi necessário o refinamento dos resultados pela leitura: primeiro, dos títulos das pesquisas, mas, quando necessário, foram consultados as palavras-chave e os resumos (trabalhos anteriores à Plataforma Sucupira $^{4}$ foram buscados externamente, nas bibliotecas digitais das instituições). Foram eliminadas as pesquisas que apresentavam moda em sentidos diferentes do tratado pela presente pesquisa (por exemplo, em casos de uso da expressão "à moda de", ou da palavra "modalidade").

Tendo organizado as informações encontradas em planilha do Excel, foi realizada uma análise quantitativa prévia das 238 teses e dissertações resultantes, entre 1987 e 2018. A experiência e os resultados dessa análise definiram o recorte no período entre 2006 e 2018, em que a produção acadêmica sobre moda na Comunicação mostrou crescimento e regularidade, então, delimitamos o período para a análise da evolução do objeto pelo critério de produção.

Neste recorte, os trabalhos de diferentes regiões do país puderam ser encontrados em repositórios digitais, garantindo a qualidade dos dados. 
Trabalhos que não puderam ser acessados e arquivados - aqueles cujos autores não autorizaram a publicação em repositórios on-line - foram eliminados do corpus de análise.

Por fim, o corpus foi definido em 173 documentos. Os dados continuaram organizados em planilha do Excel e os arquivos dos trabalhos completos foram salvos, identificados numericamente de acordo com a planilha, e armazenados para as próximas etapas da pesquisa.

Os indicadores definidos para as análises apresentadas a seguir foram:
a) autoria;
b) título;
c) instituição de ensino superior (IES);
d) estado (UF);
e) tipo IES (Pública/Privada);
f) nível (Mestrado/Doutorado);
g) ano de defesa;
h) orientação;
i) palavras-chave.

A interpretação dos dados combinou a análise fornecida pelos softwares Iramuteq (utilizado na análise das palavras-chave) e RStudio (utilizado para analisar os demais indicadores) com o referencial teórico e reflexivo discutido neste artigo.

\section{Discussão dos resultados}

Durante o levantamento de informações para a formação do corpus, observamos que entre 1987 e 1995, apenas cinco pesquisas sobre moda foram produzidas na área da comunicação. De 1997 até 2005, no entanto, o número tem um crescimento considerável, passando para 55 pesquisas apresentadas. $\mathrm{O}$ número de cursos superiores de moda cresceu a partir de 1988 e, em 2005, já eram 33 em todo o país, incluindo em instituições públicas, o que incentivou a formação de professores pesquisadores (TREVISOL NETO, 2015). 
Também em 2005, surge o primeiro evento anual e de cobertura nacional dedicado à pesquisa acadêmica em Moda: o Colóquio de Moda, cuja primeira edição aconteceu em Ribeirão Preto (SP), no Centro Universitário Moura Lacerda e reuniu 16 instituições de ensino, de nove Estados brasileiros, com 77 trabalhos apresentados 5 .

Nesse contexto, em 2006, nota-se que a produção se torna constante, variando entre no mínimo oito e, no máximo, 20 pesquisas defendidas em um mesmo ano (média de 13,3/ano). Só entre 2015 e 2017, foram defendidas 58 pesquisas, ou seja, quase o total do que foi produzido nos primeiros 18 anos supracitados.No período de maior produtividade, encontramos 31 teses e 142 dissertações.

Figura 1- Distribuição longitudinal dos estudos sobre moda na comunicação (2006-2018), entre Doutorados (D) e Mestrados (M).

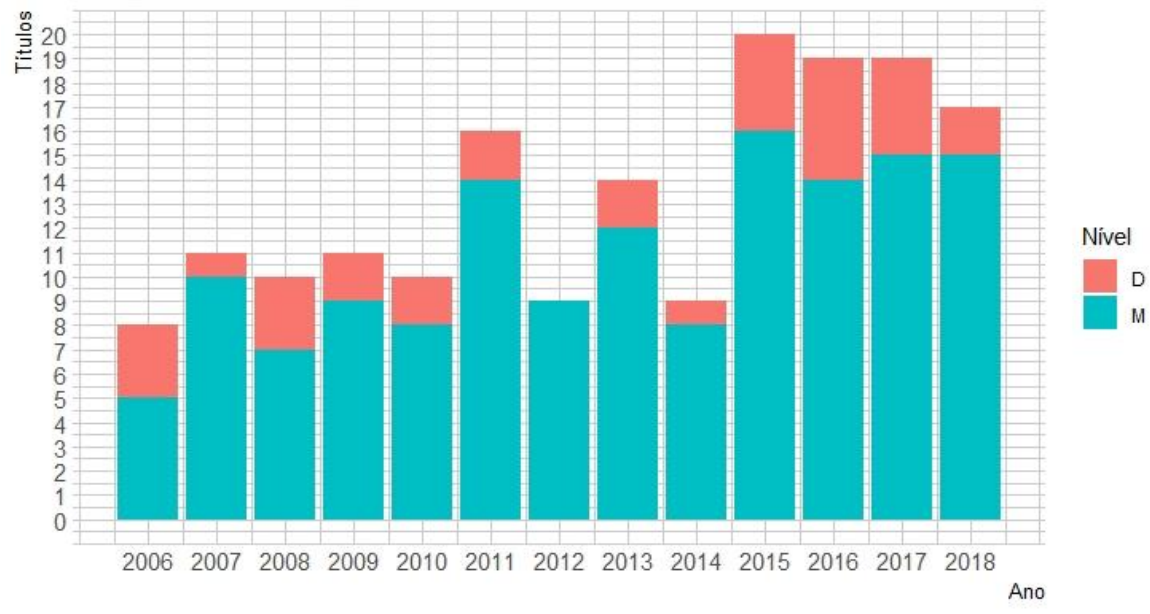

Fonte: Elaborada pelos autores (2020).

De acordo com o mesmo repositório da Capes, neste período, foram defendidas 9.985 pesquisas na área de concentração da Comunicação, sendo 7.586 dissertações e 2.308 teses. Ou seja, apesar do volume considerável, as pesquisas que compõem nosso corpus representam apenas $1.73 \%$ da produção total no intervalo de 13 anos.

A relação entre teses e dissertações na produção total da área da Comunicação é de $76 \%$ de dissertações de mestrado $(n=7.586)$ para $24 \%$ de teses de doutorado $(n=2.308)$, enquanto na produção sobre moda essa relação 
passa para $82 \%$ de dissertações de mestrado $(n=142)$ e $18 \%$ de teses de doutorado $(n=31)$. Muito autores passaram pelo tema da moda durante o mestrado, mas, não continuaram suas pesquisas sobre este objeto, se relacionando com o tema de forma ocasional.

Cabe citar como fato observável que apenas duas pessoas neste conjunto de trabalhos fizeram mestrado e doutorado sobre o tema: Mário Queiroz Antônio Pinto (Pontifícia Universidade Católica-SP) e Rosane Feijão de Toledo Camargo (Pontifícia Universidade Católica - RJ e Universidade Estadual do Rio de Janeiro).

Figura 2 - Distribuição da produção por estado e IES.

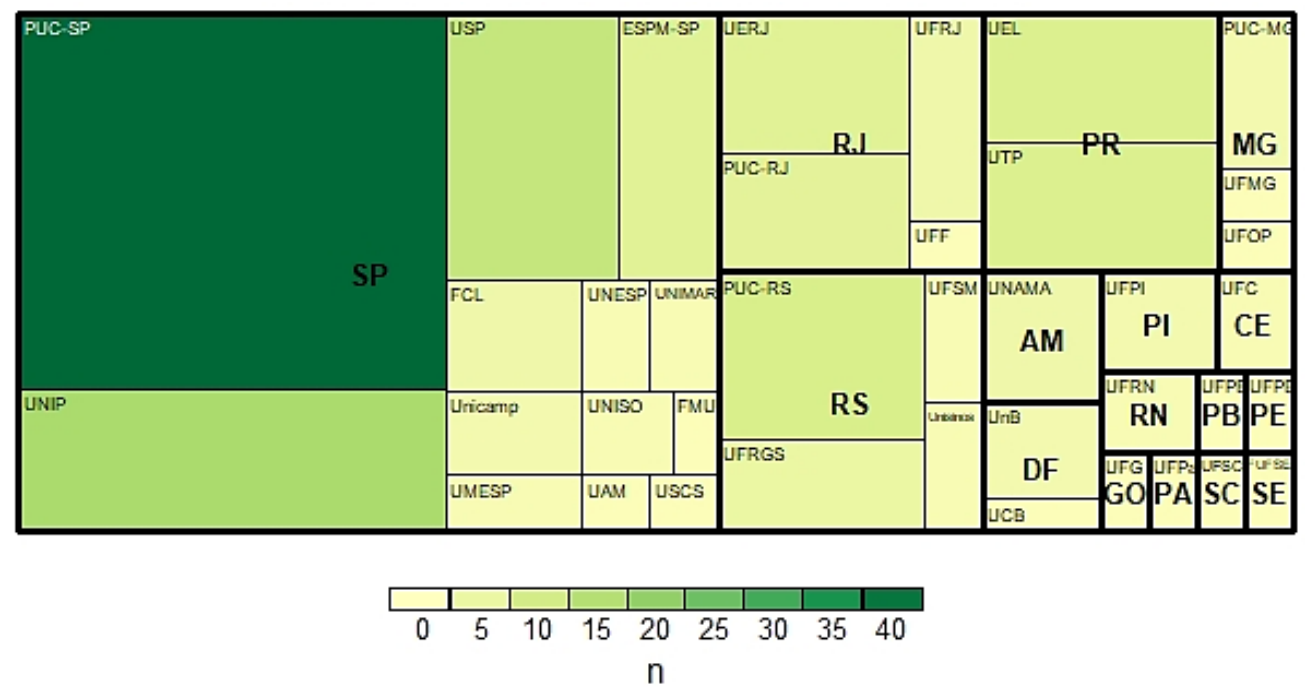

Fonte: Elaborada pelos autores (2020).

Apesar da presença de IES de todas as regiões do país, com 16 Estados representados, as regiões Sudeste e Sul concentram a maior parte da produção, com 118 e 35 pesquisas defendidas, respectivamente. O Estado de São Paulo $(\mathrm{n}=95)$ se destaca, principalmente, pela presença da Pontifícia Universidade Católica (PUC-SP).

Com 42 pesquisas compondo o corpus de análise, pudemos constatar que a IES se sobressai como centro nucleador do conhecimento acadêmico sobre moda no Brasil, seguido pela Universidade Paulista (UNIP), com 16 títulos, e pela Universidade de São Paulo (USP), com 12. Esta concentração nas IES paulistanas era esperada, dado o cenário socioeconômico e a relevância da moda 
como indústria e mercado de consumo na região. Porém, tal discrepância nos números é digna de aprofundamento e será explorada de forma qualitativa no decorrer da pesquisa.

Ao todo, 38 IES apareceram nos resultados, destacando-se ainda a Pontifícia Universidade Católica do Rio Grande do Sul (PUC-RS, n=9), a Universidade Estadual de Londrina (UEL, $\mathrm{n}=8$ ), a Universidade Tuiuti do Paraná (UTP, n=8), a Escola Superior de Propaganda e Marketing, em São Paulo (ESPM-SP, n=7), a Universidade do Estado do Rio de Janeiro (UERJ, n=7), a Pontifícia Universidade Católica do Rio de Janeiro (PUC-RJ, n=6), e a Universidade Federal do Rio Grande do Sul (UFRGS, n=5). A produção fora do eixo Sul-Sudeste apresenta crescimento apenas a partir de 2012, com destaque para a Universidade da Amazônia (UNAMA, n=4).

Ainda estão presentes: Faculdade Cásper Líbero (FCL, n=4), Universidade Federal do Rio de Janeiro (UFRJ, n=4), Pontifícia Universidade Católica de Minas Gerais (PUC-MG), Universidade Federal do Piauí (UFPI, $\mathrm{n}=3$ ), Universidade de Brasília $(\mathrm{UnB}, \mathrm{n}=3)$ e Universidade Estadual de Campinas (Unicamp, $\mathrm{n}=3$ ).

Com dois documentos, estão Universidade Federal do Ceará (UFC), Universidade Federal do Rio Grande do Norte (UFRN), Universidade Federal de Santa Maria (UFSM), Universidade Metodista de São Paulo (UMESP), Universidade Estadual Paulista (UNESP), Universidade de Marília (UNIMAR), Universidade do Vale do Rio dos Sinos (Unisinos) e Universidade de Sorocaba (UNISO).

Com apenas um documento, estão: Faculdades Metropolitanas Unidas (FMU/FIAM-FAAM), Fundação Universidade Federal do Sergipe (FUFSE), Universidade Anhembi Morumbi (UAM), Universidade Católica de Brasília (UCB), Universidade Federal Fluminense (UFF), Universidade Federal de Goiás (UFG), Universidade Federal de Minas Gerais (UFMG), Universidade Federal de Ouro Preto (UFOP), Universidade Federal do Pará (UFPa), Universidade Federal da Paraíba (UFPB), Universidade Federal de Pernambuco (UFPE), Universidade Federal de Santa Catarina (UFSC) e Universidade Municipal de São Caetano do Sul (USCS). 


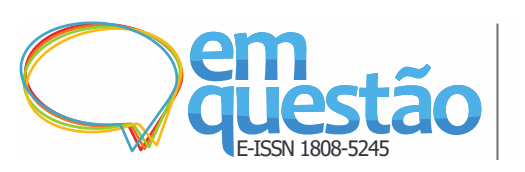

Estudo bibliométrico sobre moda em dissertações e teses da área de Ciências da Comunicação

Eneus Trindade Priscila Rezende Carvalho e Francisco Carlos Paletta

Figura 3 - Distribuição por tipo de IES.

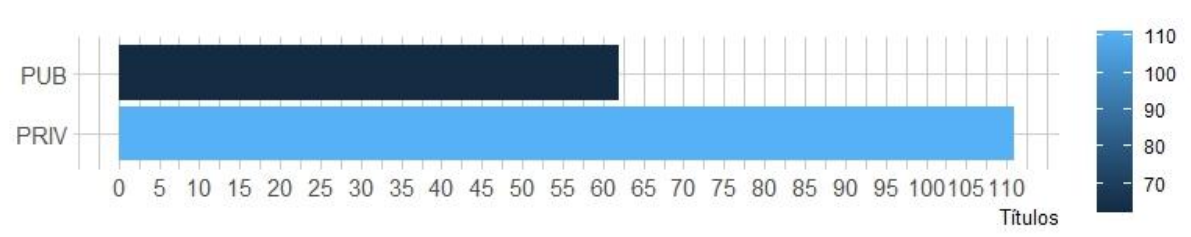

Fonte: Elaborada pelos autores (2020).

Impactada pelos números da PUC-SP, a distribuição entre instituições públicas e privadas revela que menos da metade da produção analisada é oriunda de IES públicas $(n=62)$. Tal resultado também desperta questionamentos, pois, no Brasil, as instituições públicas são responsáveis pela maior parte da produção científica e acadêmica ${ }^{6}$ (RESEARCH, 2019).

Tradicionalmente, as escolas e os cursos superiores de moda são criados sustentados por princípios do mercado, da indústria têxtil e de confecções, pautados pela demanda por profissionais qualificados. Isso explica, em parte, a maior abertura para a disciplina em instituições privadas do que em instituições públicas. O fato de a PUC-SP ter a maior produção desvela, no entanto, outra discussão: a instituição não tem cursos de graduação (ou similares) em moda. A ligação com o tema está centralizada na pós-graduação, no programa de Comunicação e Semiótica, com fins acadêmicos e científicos. A explicação para tal destaque está no grupo de pesquisadores que o programa reúne.

Nas IES mencionadas, a análise apontou 106 orientadores diferentes. Entre eles, 79 orientaram apenas um trabalho sobre moda, 16 orientaram dois trabalhos e apenas 11 orientaram mais de três trabalhos no período da pesquisa. A Profa. Dra. Ana Claudia Mei Alves de Oliveira, da PUC-SP, se destaca com 18 orientações, seguida da Profa. Dra. Solange Wanjman (UNIP, n=7), do Prof. Dr. Norval Baitello Junior (PUC-SP, n=6) e da Profa. Dra. Leda Tenório da Motta (PUC-SP, n=5).

Percebemos, portanto, que o destaque da PUC-SP é resultado da atuação de vários pesquisadores envolvidos com o tema de forma constante, e não esporádica. A institucionalização social da moda na comunicação, portanto, evidencia concentrações e uma pequena comunidade acadêmica, sendo a PUCSP a que reúne maior número de agentes. 
Figura 4 - Pesquisadores com maior volume de orientações.

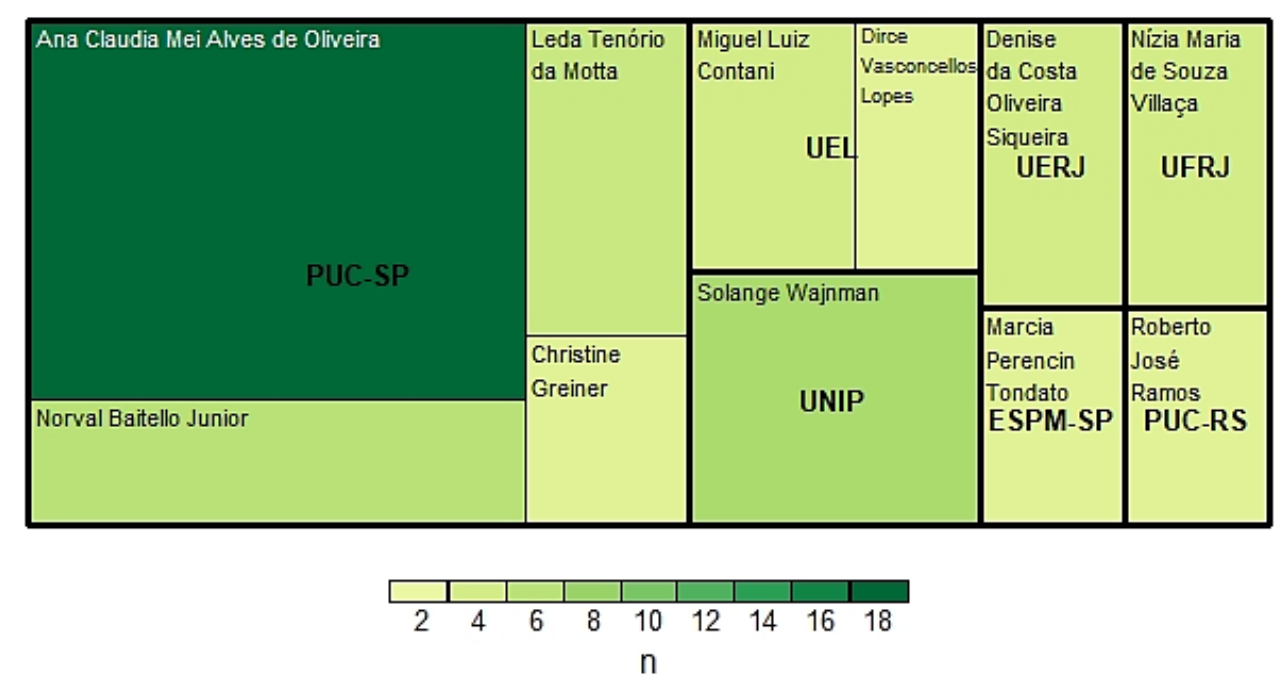

Fonte: Elaborada pelos autores (2020).

Entre os outros pesquisadores que contribuem com a interface estão: Denise da Costa Oliveira Siqueira (UERJ), Miguel Luiz Contani (UEL) e Nízia Maria de Souza Villaça (UFRJ), com $n=4$ cada. Christine Greiner (PUC-SP), Dirce Vasconcellos Lopes (UEL), Marcia Perencin Tondato (ESPM-SP) e Roberto José Ramos (PUC-RS), com n=3 cada.

Carla Reis Longhi (UNIP), Dulcília Helena Schroeder Buitoni (FCL), Eneus Trindade Barreto Filho (USP), Ernesto Giovanni (Unicamp), Everardo Pereira Rocha (PUC-RJ), José Salvador Faro (UMESP), Lucia Isaltina Clemente Leão (PUC-SP), Mágda Rodrigues da Cunha (PUC-RS), Maria Clotilde Perez Rodrigues (USP), Maria Lucia Santaella (PUC-SP), Michael Manfred Hanke(UFRN), Sandra Fischer (UTP), Sandra Rúbia da Silva (UTP) e Tania Marcia Cezar Hoff (ESPM-SP), com n=2 cada.

Com relação às temáticas estudadas no encontro entre comunicação e moda, recorremos às palavras-chave para enxergar, no excesso de dados e de informação, tendências possíveis de estudos que caracterizam o conhecimento produzido. Para tanto, todas as palavras-chave do corpus de análise foram agrupadas por ano e submetidas à análise de similitude, uma análise que possibilita identificar as coocorrências entre as palavras, indicando as conexões entre as mais frequentes (CAMARGO; JUSTO, 2013). 
Figura 5 - Análise de similitude das palavras-chave.

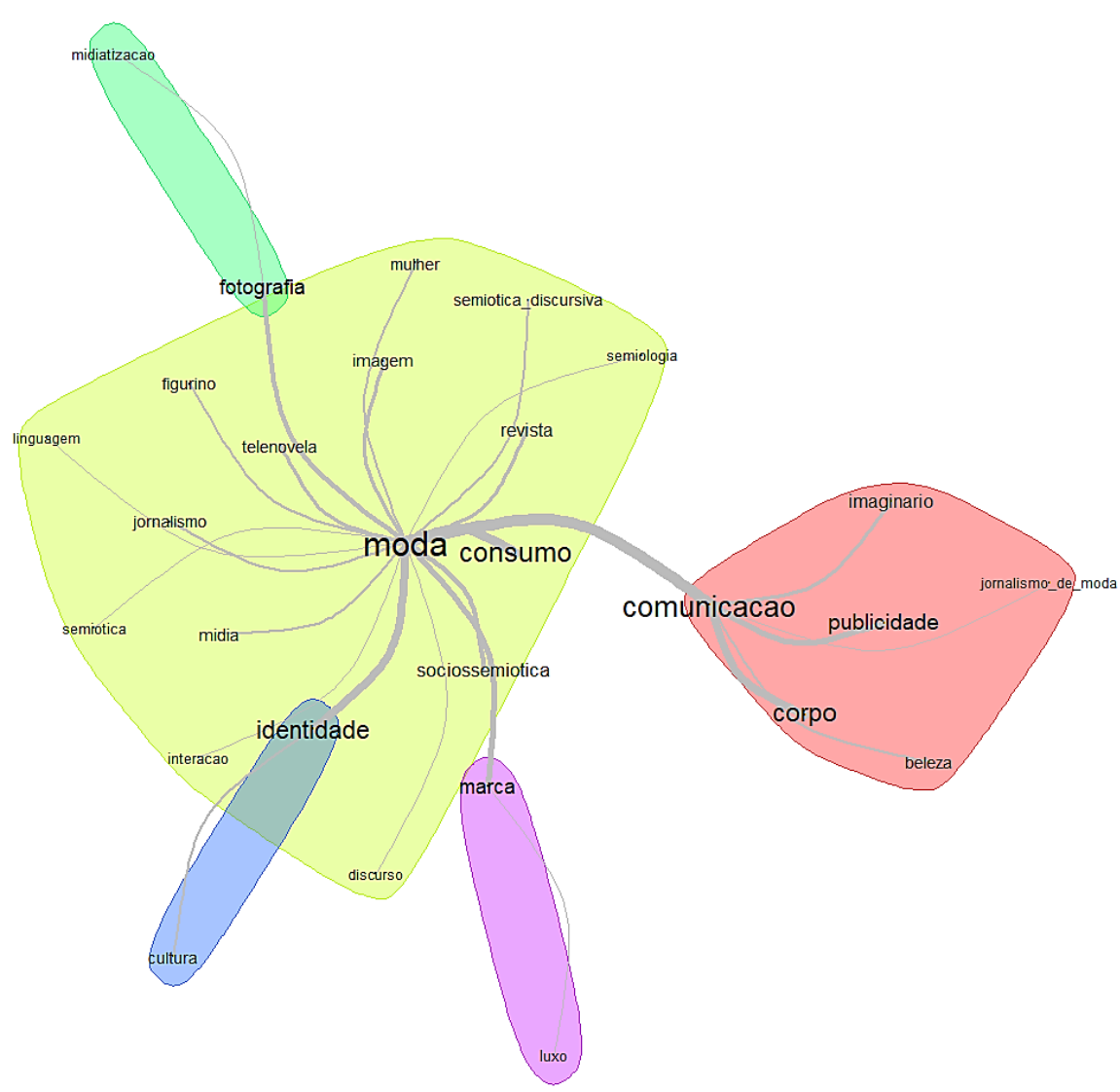

Fonte: Elaborada pelos autores (2020).

Foram selecionadas 28 palavras mencionadas pelo menos quatro vezes, ao todo, 326 ocorrências. Dessa forma, mitigamos a dispersão temática e o foco recai nas palavras com maior potencial de indicar tendências, otimizando a visualização da informação.

Como era esperado, moda $(n=82)$, comunicação $(n=36)$ e consumo $(n=27)$ são as três palavras mais frequentes. A partir delas, podem ser visualizados agrupamentos lexicais representativos da produção de conhecimento analisada.

O agrupamento central, em que se destacam os termos "moda" e "consumo", indica a predominância de metodologias voltadas para os estudos semióticos e do discurso sobre produtos midiáticos segmentados para o público 
feminino, inferência feita a partir da palavra "mulher" entre os termos aproximados e constatada pela leitura dos títulos e dos resumos.

O agrupamento destacado pelos termos "comunicação", "publicidade" e “corpo" revela que as prescrições acerca dos ideais relacionados ao corpo são mais debatidas nessa interface do que os bens de consumo como o vestuário, propriamente.

Os outros agrupamentos trazem conexões mais objetivas entre "marca" e "luxo"; "identidade" e "cultura"; e "midiatização" e "fotografia", indicando linhas de investigação exploradas e contribuições da moda como objeto para o campo da Comunicação em interface com o consumo. Nesses agrupamentos, enxergamos tendências de pesquisa que caracterizam o que já foi produzido, dando a entender o estado atual do conhecimento, mas que, além disso, envergam os sentidos da moda em várias direções, revelando a versatilidade do tema para pesquisas futuras.

\section{Considerações finais}

Com estas análises, buscamos demonstrar a dimensão da produção científica e acadêmica sobre moda na área da Comunicação e constatamos que o número de pesquisas cresceu nos últimos anos, com forte relação com os estudos dos consumos. Por meio de técnicas bibliométricas e de organização e visualização da informação, percebemos, de forma inicial, as características desta produção: representada, em sua maioria por dissertações de mestrado, realizadas em instituições privadas nas regiões Sudeste e Sul, que objetivam produtos midiáticos voltados para o segmento feminino, marcas de luxo, identidade e cultura e a fotografia como midiatização. Cada um desses agrupamentos aponta trajetos para análises qualitativas futuras.

A hipótese proposta não se confirmou: apesar de apresentar dispersões por áreas e objetos múltiplos, quando focamos no campo da Comunicação, percebemos que a moda tem se institucionalizado de forma concentrada. No âmbito social, vimos que poucos pesquisadores e instituições de ensino superior são responsáveis pelo maior volume de pesquisas defendidas, o que reflete no 
aspecto cognitivo, em que a maior parte das pesquisas se referem a estudos semióticos realizados, principalmente, pela PUC-SP.

Aplicada como técnica exploratória nas Ciências da Comunicação, a bibliometria conduziu a resultados que localizam e esboçam a historicidade do objeto, proveniente de um cenário muito específico, mostrando tendências de estudos mais estabelecidas e com potencial de amadurecimento. Para futuros estudos, oferecemos um mapeamento introdutório de agentes, de instituições e de temas que se referem aos processos interacionais da moda pelo consumo.

Para o prosseguimento desta pesquisa, ficaram definidos o corpus de análise e os questionamentos decorrentes, que deverão ser desenvolvidos em uma imersão qualitativa na informação produzida: quais os saberes sobre moda se desenvolvem em cada uma das tendências levantadas? Quais relações contextuais são traçáveis a partir dos resultados quantitativos e dos indícios sobre a concentração da produção? Quais as confluências entre a moda tratada no campo acadêmico e a moda como fenômeno sociocultural e mercadológico no período estudado? Esses aspectos que serão explorados ao longo da tese.

Há um volume relevante de conhecimento gerado que deve ser entendido pelo seu estado da arte, fornecendo bases para novas pesquisas e, também, por sua possibilidade de indicar tendências, visando a iniciativas no campo científico, sejam projetos de pós-graduação, grupos de estudos ou eventos. Além de afirmar que há crescimento na produção, buscamos temas que apresentam oportunidades e lacunas para novas pesquisas, oferecendo pontos de partida e conhecimentos prévios.

\section{Financiamento}

O presente trabalho foi realizado com apoio da Coordenação de Aperfeiçoamento de Pessoal de Nível Superior - Brasil (CAPES) - Código de Financiamento 001. 


\section{Referências}

ALARCÓN, Eduardo Villena. La producción científica españolaen moda a través de lãs tesis doctorales. Revista Prisma Social, Madrid, n. 24, p. 209-232, 2019.

ARAÚJO, Carlos A. Bibliometria: evolução histórica e questões atuais. Em Questão, Porto Alegre, v. 12, n. 1, p. 11-32, 2006.

BARBOSA, Livia; CAMPBELL, Colin. O estudo do consumo nas ciências sociais contemporâneas. Cultura, consumo e identidade. Rio de Janeiro: Editora FGV, 2006.

BEDERSON, Benjamin B.; SHNEIDERMAN, Ben. The craft of information visualization: readings and reflections. Burlington: Morgan Kaufmann, 2003.

BONADIO, Maria Claudia. A produção acadêmica sobre moda na pósgraduação stricto sensu no Brasil. Iara - Revista de Moda, Cultura e Arte, São Paulo, v.3, n. 3, p. 50 - 146, dez. 2010.

BORGMAN, Christine. Bibliometrics and Scholarly Communication.

Communication Research, California, p. 583-599, Oct. 1989.

BORGMAN, Christine; FURNER, Jonathan. Scholarly Communication and Bibliometrics. Annual Review of Information Science and Technology, USA, v. 36, n. 1, p. 3 - 72, Feb. 2002.

BOURDIEU, Pierre, O campo científico. In: ORTIZ, Renato (org.). Pierre Bourdieu. São Paulo: Ática, 1983.

BOURDIEU, Pierre; CHAMBOREDON, Jean-Claude; PASSERON, JeanClaude. A profissão de sociólogo: preliminares epistemológicas. Petrópolis: Vozes, 1999.

BRAGA, José Luiz. Os estudos de interface como espaço de construção do Campo da Comunicação. Revista Contracampo, Niterói, n. 10/11, p. 219-236, 2004.

CAFÉ, Ligia Maria Arruda; BRÄSCHER, Marisa. Organização da informação e bibliometria. Encontros Bibli: revista eletrônica de biblioteconomia e ciência da informação, Florianópolis, p. 54-75, jan. 2008.

CAMARGO, Brigido Vizeu; JUSTO, Ana Maria. Tutorial para uso do software de análise textual IRAMUTEQ. Universidade Federal de Santa Catarina, 2013.

CHEN,Chaomei. Informationvisualization: beyond the horizon. Springer: Verlag London, 2006. 
CÔRTES, Pedro Luiz; RODRIGUES, Rosely. A bibliometric study on "education for sustainability". Brazilian Journal of Science and Technology, [s.l.], v. 3, n. 1, p. 8, 2016.

FUNARO, Vânia Martins Bueno de Oliveira; NORONHA, Daisy Pires. Literatura cinzenta: canais de distribuição e incidência nas bases de dados. In: Comunicação e produção científica: contexto, indicadores, avaliação. Dinah Aguiar Poblacion, Geraldina Porto Witter, José Fernando Modesto da Silva (org.). São Paulo: Angellara, 2006.

KOBASHI, Nair Yumiko; DOS SANTOS, Raimundo Nonato Macedo. Arqueologia do trabalho imaterial: uma aplicação bibliométrica à análise de dissertações e teses. Encontros Bibli: revista eletrônica de biblioteconomia e ciência da informação, Florianópolis, v. 13, n. 1, p. 106-115, 2008.

LARA, Marilda Lopes Ginaz. Termos e conceitos da área de comunicação e produção científica. In: Comunicação e Produção Científica: Contextos, Indicadores e Avaliação. Dinah Aguiar Poblacion, Geraldina Porto Witter, José Fernando Modesto da Silva (org.). São Paulo: Angellara, 2006.

LOPES, Maria ImmacolataVassallo de. Sobre o estatuto disciplinar do campo da comunicação. Epistemologia da comunicação. São Paulo: Loyola, 2003.

LOPES, Maria ImmacolataVassallo de. Reflexividade e relacionismo como questões epistemológicas na pesquisa empírica em comunicação. In: Pesquisa empírica em comunicação. José Luiz Braga, Maria ImmacolataVassallo de Lopes, Luiz Claudio Martino (org.).São Paulo: Paulus, 2010.

LOPES, Sílvia et al. A Bibliometria e a Avaliação da Produção Científica: indicadores e ferramentas. In: Actas do congresso Nacional de bibliotecários, arquivistas e documentalistas. Lisboa: Associação Portuguesa de Bibliotecários, Arquivistas e Documentalistas, 2012.

MARICATO, João de Melo; NORONHA, Daysi Pires. Indicadores bibliométricos e cientométricos em CT\&I: apontamentos históricos, metodológicos e tendências de aplicação. In: Bibliometria e cientometria: reflexões teóricas e interfaces. Maria Christina Piumbato Innocentini.Hayashi et al.(org.). São Carlos: Pedro \& João Editores, 2013.

McCRACKEN, Grant.Cultura \& Consumo: novas abordagens ao caráter simbólico dos bens e atividades de consumo. Rio de Janeiro: Mauad, 2003. OLIVEIRA, Paulo Felipe de; GUERRA, Saulo; MCDONNELL,Robert.Ciência de dados com R: Introdução. Brasília: Editora IBPAD, 2018.

PERES-NETO, Luiz. Teorias da comunicação e o consumo: algumas conjecturas teóricas e prospecções. In: Encontro Anual daCompós, 25., Goiânia, Anais [...]. Universidade Federal de Goiás: GT Consumos e Processos de Comunicação, 2016. 
PRADO, Luís André. Gilda de Mello e Souza e a emergência do campo da moda no Brasil (1800-1990). Revista de História, São Paulo, n. 178, p. 1-31, 2019.

QUEVEDO-SILVA, Filipe et al. Estudo bibliométrico: orientações sobre sua aplicação. Revista Brasileira de Marketing, São Paulo, v. 15, n. 2, p. 246-262, 2016.

RAINHO, Maria Do Carmo Teixeira; VOLPI, Maria Cristina. Looking at Brazilian fashion studies: Fifty years of research and teaching. International Journal of Fashion Studies, London, v. 5, n. 1, p. 211-226, 2018.

RESEARCH IN BRAZIL: Funding excellence. Analysis prepared on behalf of CAPES by the Web of Science Group. Jornal da USP, São Paulo, 05 set. 2019.

SILVA, Edna Lucia da; CAFÉ, Ligia Maria Arruda; TREVISOLNETO, Orestes. A institucionalização científica do campo da moda no Brasil. ECCOM, Lorena, v. 8, n. 15, p. 133 - 152, jan./jun. 2017.

SODRÉ, Muniz. A ciência do comum. Petrópolis: EditoraVozes, 2014.

TREVISOL NETO, Orestes. A institucionalização científica do campo da Moda no Brasil: estudo baseado nas instituições, produtores e produtos científicos. 2015. 191 f. Dissertação (Mestrado em Ciência da Informação) -Universidade Federal de Santa Catarina, Florianópolis, 2015.

TRINDADE, Eneus. Tendências sobre publicidade e consumo em revistas científicas da comunicação Qualis A2 entre 2006 e 2017. Signos do Consumo, São Paulo, v. 11, n. 2, p. 114-125, 2019.

WHITLEY, Richard. Cognitive and social institucionalization of scientific specialities and research. In: WHITLEY, Richard (ed.). Social process of scientific development. London: Routledge and Kegan, 1974.

YALE, Laura., GILLY, Mary C. Trends in Advertising Research: A Look at the Content of marketing-Oriented Journalsfrom 1976 to 1985. Journal of Advertising, USA, v. 17, n. 1 p. 12-22, 1988.

\title{
Bibliometric study on theses and dissertations about fashion in
}

\section{Communication Sciences.}

\begin{abstract}
The recent institutionalization of fashion as a scientific and academicfield, and the growing interest on it observed in a range of areas, are the assumptions in this bibliometric study on theses and dissertations. The aim is
\end{abstract}


to scale and characterize the production in postgraduate programs in Communication Sciences in Brazil. It explores quantitativelyhow this area of studies, particularly, has fomented the debate on fashion in the academic scope.Therefore, three main topics are discussed: the relation between fashion and consumer studies in Communications, bibliometrics and information organization, and the uses of computational tools for visualizing and communicating results. The time period analyzed is from 2006 to 2018, which shows the increasing production about the subject. Beyond demonstrating the evolution, the results highlight the institutions and the researchers with more contributions, and relate the most used keywords, glimpsing tendencies of research.

Keywords: Information Organization. Bibliometrics. Theses and Dissertations. Communication. Fashion.

Recebido: 07/08/2020

Aceito: 19/01/2021

\section{Declaração de autoria}

Concepção e elaboração do estudo: Eneus Trindade, Priscila Rezende Carvalho, Francisco Carlos Paletta

Coleta de dados: Priscila Rezende Carvalho, Eneus Trindade, Francisco Carlos Paletta

Análise e interpretação de dados: Priscila Rezende Carvalho, Eneus Trindade, Francisco Carlos Paletta

Redação: Priscila Rezende Carvalho, Eneus Trindade, Francisco Carlos Paletta

Revisão crítica do manuscrito: Eneus Trindade, Francisco Carlos Paletta, Priscila Rezende Carvalho

\section{Como citar}

TRINDADE, Eneus; CARVALHO, Priscila Rezende; PALETTA, Francisco Carlos. Estudo bibliométrico sobre moda em dissertações e teses da área de Ciências da Comunicação. Em Questão, Porto Alegre, v. 27, n. 3, p. 251-274, 2021, Doi: http://dx.doi.org/10.19132/1808-5245273.251-274

\footnotetext{
${ }^{1}$ Entre 2004 e 2010, o Centro Universitário Senac ofereceu o mestrado em Moda, Cultura e Arte, onde foram defendidas 39 dissertações, de acordo com Maria Claudia Bonadio (2010).

2 "O IRAMUTEQ é um software gratuito e com fonte aberta, desenvolvido por Pierre Ratinaud", para mais informações e acesso ao software e à comunidade de desenvolvedores e usuários: http://www.iramuteq.org/documentation/fichiers/tutoriel-en-portugais.

${ }^{3}$ Interface disponível em versões gratuita ou paga, com ferramentas abertas e comunidades de desenvolvedores em diversos países: https://rstudio.com/about/

${ }^{4}$ A Plataforma Sucupira foi lançada em 2014:https://www.capes.gov.br/avaliacao/plataformasucupira.
} 
${ }^{5}$ Informação disponível em: http://coloquiomoda.com.br/coloquio2013/1-coloquio-demoda.php. Última visita em: 08-01-2021.
${ }^{6}$ Informação
disponível
em:
https://jornal.usp.br/wpcontent/uploads/2019/09/ClarivateReport_2013-2018.pdf. Acesso em: 16-06-2020. 